\title{
Symbiotic Gene Sym31 Controls the Presence of a Lectinlike Glycoprotein in the Symbiosome Compartment of Nitrogen-Fixing Pea Nodules
}

\author{
Preeti Dahiya, D. Janine Sherrier, Igor V. Kardailsky, Alexey Y. Borisov, and Nicholas J. Brewin \\ John Innes Centre, Colney Lane, Norwich, NR4 7UH, Great Britain. \\ Accepted 12 May 1998.
}

The distribution of a lectinlike glycoprotein, PsNLEC-1, was examined with a specific antiserum in nodule tissue from a symbiotically defective pea mutant, Sprint $2 \mathrm{Fix}^{-}$ (sym31), and the parent line, Sprint2. Immunostaining of Western blots (immunoblots) revealed that, whereas wildtype nodules contained three antigenic isoforms of PsNLEC-1, nodule homogenates of mutant sym31 contained only one isoform, PsNLEC-1C. Fractionation studies indicated that PsNLEC-1C was not associated with symbiosomes in either the mutant or the wild-type parent (unlike the other two isoforms from wild-type nodules). Light microscopy revealed that PSNLEC-1 antigen was more abundant in the infected tissues of wild-type nodules than in nodules of sym31. By contrast, in situ hybridization indicated that the PsNlec1 gene transcript was strongly expressed in infected cells of both Sprint 2 and sym31 nodule tissues. At the ultrastructural level, most of the PsNLEC-1 antigen in sym31 nodule tissue was visualized as inclusion bodies in the vacuolar compartment of infected host cells but it was apparently absent from the symbiosome compartment. The results suggest an aberrant vesicle targeting pathway during symbiosome development in this mutant.

Additional keywords: Golgi body, lectin, legume, nitrogen fixation, Pisum sativum, Rhizobium leguminosarum bv. viciae.

Nitrogen-fixing root nodules are formed as a result of the symbiosis between Rhizobium spp. and leguminous plants. Establishing such a highly evolved, mutualistic interaction involves a complex sequence of morphological developments that follow the initial exchange of signal molecules between the bacteria and the host plant root (Brewin 1991; Mylona et al. 1995). Indeterminate nodules, such as those of pea (Pisum sativum L.) and alfalfa (Medicago sativa L.), show an axis of

Corresponding author: N. J. Brewin; Telephone: +44 (0) 1603 452571; Fax: +44 (0) 1603 456844; E-mail: brewin@bbsrc.ac.uk

Present address of Janine Sherrier: Department of Biochemistry, Tennis Court Road, Cambridge CB2 1QW, Great Britain.

Present address of Igor V. Kardailsky: The Salk Institute for Biological Studies, 10010 N. Torrey Pines Road, La Jolla, CA 92037, U.S.A.

Permanent address of Alexey Y. Borisov: Research Institute for Agricultural Microbiology, St. Petersburg-Pushkin, Russia. cell development along the median longitudinal axis of the nodule. There is a persistent apical meristem (zone I), followed by a prefixation zone (II), interzone (II/III), and nitrogen-fixing zone (III) (Vasse et al. 1990). In the prefixation zone, rhizobia spread through transcellular infection threads and are released into the host cytoplasm from unwalled infection droplets. The released bacteria form part of the symbiosome, a specialized compartment comprising bacteroids surrounded individually by a symbiosomal (or peribacteroid) membrane (Verma and Hong 1996). In interzone II/III, the symbiosome apparently differentiates into an $\mathrm{N}_{2}$-fixing, organelle-like structure. This transition involves changes in the composition of symbiosomal and bacteroid membranes. In pea nodules, the bacteroids stop dividing, enlarge, and become Yshaped. At the same time, there is transcriptional induction of genes encoding nitrogenase and related functions (Mylona et al. 1995). Mature, infected cells containing symbiosomes occupy zone III of the nodule in which nitrogen fixation takes place and, ultimately, symbiosomes and host cells senesce in zone IV at the base of the nodule.

Thus, the legume nodule is a highly organized tissue and the study of symbiotically defective mutants (of either symbiont) can help us to understand the processes underlying nodule formation. An interesting case is the pea mutation in Sprint2Fix ${ }^{-}$(sym31). Previous evidence indicates that the mutation in this line determines abnormal symbiosome structure and reduced morphological differentiation of bacteroids (Borisov et al. 1992). In the wild-type pea nodule cell, each symbiosome normally harbors an individual bacteroid. However, in the Sprint 2 Fix $^{-}$mutant, symbiosomes contain multiple bacteroids that are morphologically undifferentiated and lack the ability to fix nitrogen. The mutant Sprint $2 \mathrm{Fix}^{-}$was originally obtained by EMS mutagenesis of the pea line Sprint2 (Borisov et al. 1992). Genetic characterization established that the phenotype could be ascribed to a single recessive mutation and the sym 31 allele has been assigned (Borisov et al. 1997). In a physiological study it was shown (Romanov et al. 1995) that the bacteroids of this mutant were active in respiration and the central tissue of the nodule maintained a low $\mathrm{O}_{2}$ environment. However, reduced levels of leghemoglobin, glutamine synthase, and sucrose synthase were observed. Although the level of starch and sucrose remained unchanged, the ononitol concentration was 15 times less in the mutant nodules, suggesting an altered carbon metabolism in sym 31 (Romanov et al. 1995). 
In a recent immunocytological study of sym31 (Sherrier et al. 1997), it was shown that a plasmalemma glycolipid antigen recognized by monoclonal antibody JIM18 was present on symbiosomal membrane of host cells throughout the nodule. Because JIM18 antigen is normally a marker for juvenile symbiosomal membranes (Perotto et al. 1995), the uniform distribution of JIM18 antigen on symbiosomal membranes of sym31 suggested that the development of the symbiosomes was blocked at an early stage of development. Furthermore, small bacteroids that were not bounded by symbiosomal membrane were sometimes observed in vacuolelike compartments. In the same study (Sherrier et al. 1997), it was also noted that bacteroids isolated from sym 31 symbiosomes failed to show the presence of lipopolysaccharide epitopes normally expressed by wild-type bacteroids prior to the induction of nitrogenase. These observations suggested that, in sym31, symbiosome development was arrested at an early stage in contrast to the mutant E135f (sym13), a symbiotically defective pea line in which symbiosome development is apparently normal but where symbiosomes senesce prematurely (Kneen et al. 1990a). Moreover, by construction of the double mutant, sym13sym31, it was shown that the wild-type allele Sym31 functions earlier in symbiosome development than Sym13 because the ultrastructural phenotype of the double mutant was indistinguishable from that of sym31 alone (Borisov et al. 1997). All these features make sym31 an interesting mutant in which to examine the targeting of Golgi-derived vesicles to the symbiosome compartment, and the glycoprotein PsNLEC-1 (Dahiya et al. 1997) now provides a suitable marker for such a study.

In a previous study (Kardailsky et al. 1996), a nodulespecific transcript expressed in pea nodule cells was isolated as a cDNA clone. On the basis of DNA sequence analysis, the corresponding gene was designated as PsNlecl, a hitherto unknown member of the legume lectin gene family that was strongly expressed in infected cells associated with pea nodule zones III and IV (Brewin and Kardailsky 1997). A specific

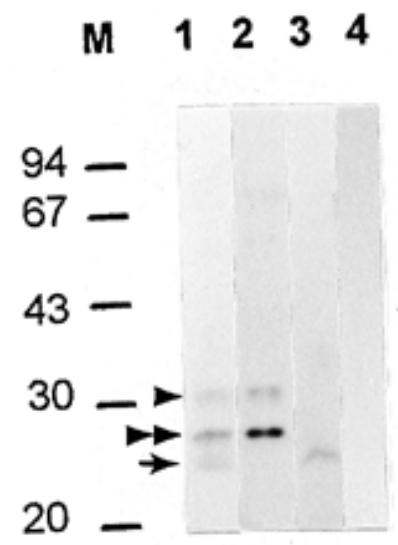

Fig. 1. Distribution of PsNLEC-1 isoforms after separation by sodium dodecyl sulfate-polyacrylamide gel electrophoresis (SDS-PAGE) from homogenates of wild-type and mutant pea nodules and peribacteroid fluid derived from isolated symbiosomes. Western blot (immunoblot) treated with R76 antiserum, followed by immunophosphatase staining. Lanes 1 and 2, Sprint2; lanes 3 and 4, sym31. Lanes 1 and 3, nodule homogenate; lanes 2 and 4, peribacteroid fluid. M, Molecular mass markers $(\times 1,000)$. Positions of 32-kDa, 27-kDa, and 25-kDa PsNLEC-1 antigenic isoforms are marked by arrowhead, double arrowhead, and arrow, respectively. antiserum was generated for PsNLEC-1 (Dahiya et al. 1997), and immunostaining of Western blots (immunoblots) indicated that two antigenic isoforms, PsNLEC-1A and PsNLEC-1B, were present in isolated symbiosomes. In nodule homogenates from pea var. Wisconsin Perfection, the antiserum also recognized an additional fast-migrating band, designated as isoform PsNLEC-1C. Fractionation studies indicated that PsNLEC-1C was associated with a $100,000 \times g$ nodule membrane fraction, suggesting an association with cytoplasmic membranes or vesicles. Preliminary analysis of the oligosaccharide sidechain of isoform 1C (L.-L. Guldemann, D. Ashford, and N. J. Brewin, unpublished results) indicated that it probably corresponds to the N-linked heptasaccharide commonly associated with vacuolar glycoproteins (Faye et al. 1989). Furthermore, using several monoclonal antibodies that recognize the carbohydrate epitopes of these Golgi-derived glycoproteins, it was shown that isoforms $1 \mathrm{~A}$ and $1 \mathrm{~B}$ (recognized by MAC266 antibody) apparently had a more complex oligosaccharide sidechain than that of isoform 1C (Dahiya et al. 1997). Immunogold localization studies also demonstrated that PsNLEC-1 antigen was present in the symbiosome compartment and, to a lesser extent, in the vacuole of host cells.

We report here the altered localization and processing of PsNLEC-1 in host cells of Sprint2Fix ${ }^{-}$(sym31). We have used the antiserum raised against PsNLEC-1 protein (Dahiya et al. 1997) to demonstrate the absence of the antigen from the

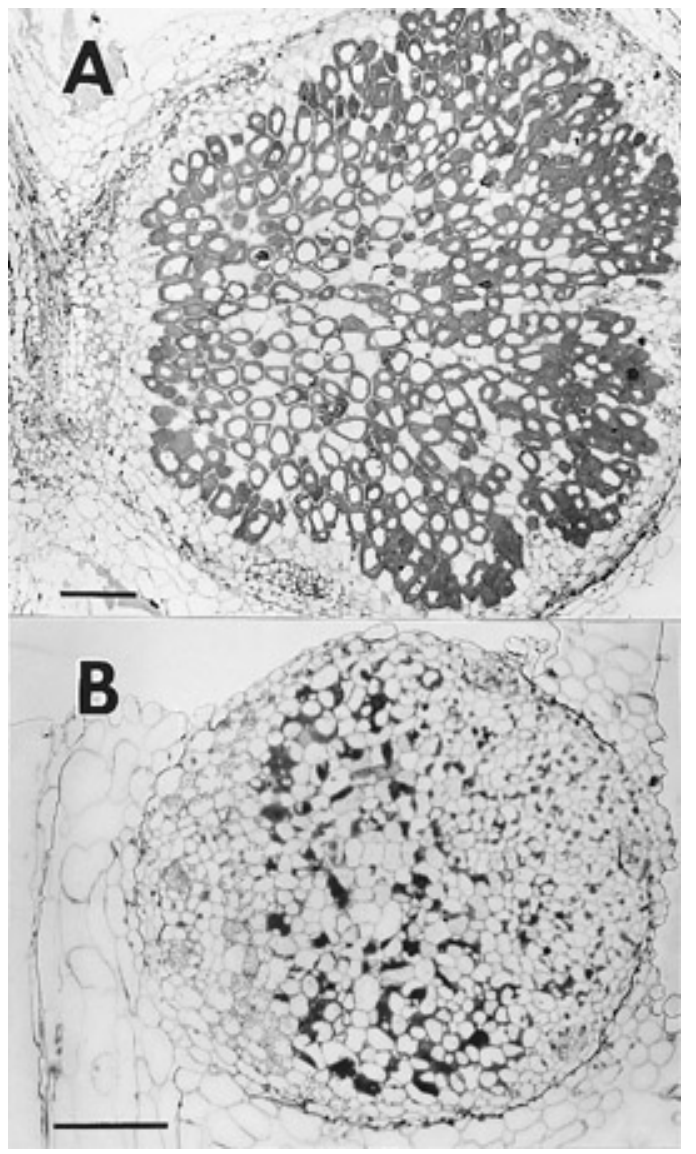

Fig. 2. Longitudinal sections of pea nodules stained with basic fuschin. A, Sprint2; B, sym31, in each case with the nodule apex oriented to the right hand side. Bar $=80 \mu \mathrm{m}$. 
symbiosomal compartment of sym31 host cells, although PsNlecl transcript was expressed by all infected cells. We also show that vacuoles of infected cells of sym 31 accumulate the PsNLEC-1 antigen, possibly as a result of defective targeting to the symbiosome compartment.

\section{RESULTS}

\section{Analysis of PsNLEC-1 antigenic isoforms}

in pea nodule homogenates.

Antiserum R76, reacting with PsNLEC-1, was used to study the distribution of antigenic isoforms in the nodules of the Sprint 2 wild-type pea and Sprint2Fix ${ }^{-}($sym 31$)$. In the total nodule homogenate of Sprint2 (Fig. 1, lane 1), three antigenic isoforms of PsNLEC-1 (isoforms A, B, and C) were observed. Isoforms PsNLEC-1A (molecular mass $32 \mathrm{kDa}$ ) and PsNLEC1B $(27 \mathrm{kDa})$ were present in the peribacteroid fluid derived from isolated symbiosomes (Fig. 1, lane 2). PsNLEC-1C (25 $\mathrm{kDa})$ was associated with nodule supernatant and cytoplasmic membrane fractions but was absent from any symbiosomal fraction, either peribacteroid fluid (Fig. 1, lane 2) or symbiosomal membrane (data not shown). Whereas the homogen-

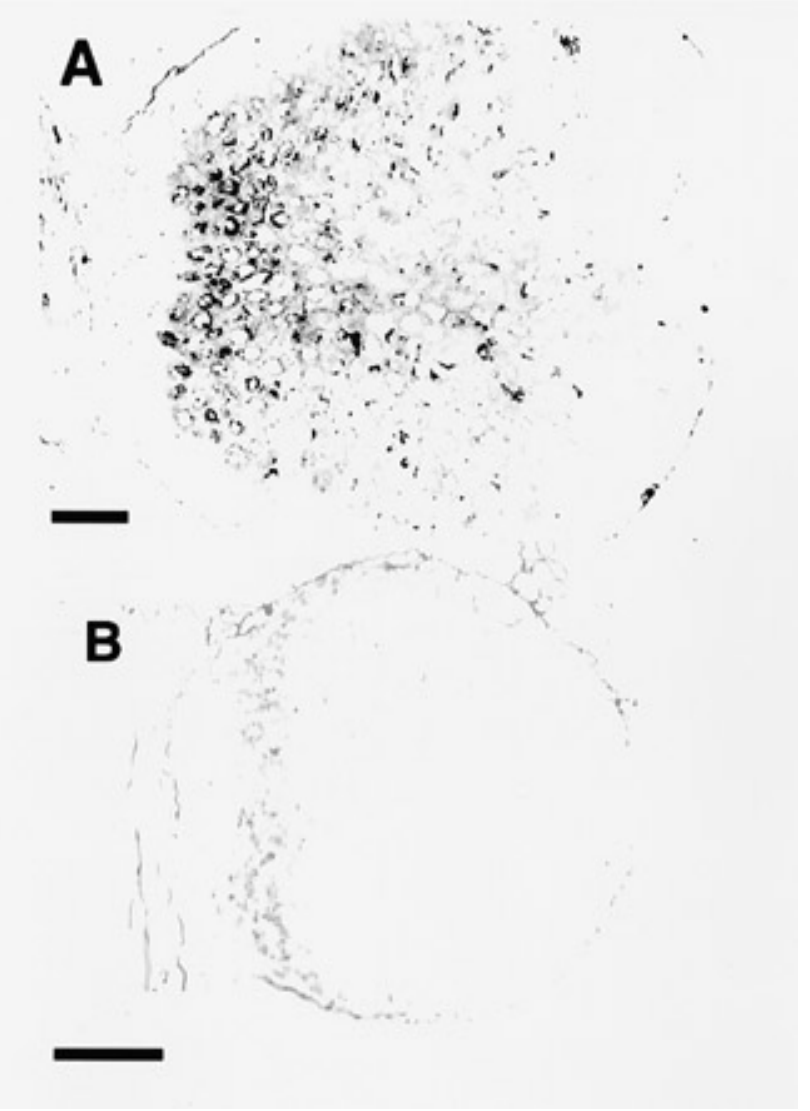

Fig. 3. Immunogold localization with silver enhancement for sections adjacent to those illustrated in Figure 2 (but without counterstaining). A, Longitudinal section of a Sprint2 nodule treated with R76 antiserum, showing a gradual increase in abundance of the PsNLEC-1 antigen toward the base of the nodule. B, sym 31 nodule section, showing undetectable immunolabeling: dark image toward base of nodule corresponds to a zone of starch granules. Other birefringent images correspond to endodermal sheath (encircling nodule) and xylem cells in the longitudinal axis of the root on left hand side of micrograph. Bar $=80 \mu \mathrm{m}$. ate of Sprint 2 wild-type nodules revealed three antigenic isoforms of PsNLEC-1, homogenate from the Fix ${ }^{-}$mutant sym31 revealed only one isoform, the nonsymbiosomal PsNLEC-1C (Fig. 1, lane 3). Furthermore, in the peribacteroid fluid derived from isolated symbiosomes of sym31, PsNLEC-1 antigen was completely undetectable (Fig. 1, lane 4).

In order to study the occurrence of PsNLEC-1 antigenic isoforms in $\mathrm{Fix}^{-}$mutants, total nodule homogenates of five symbiotically defective mutants (sym13, sym31, FN1, E107, and sym26) were analyzed on immunoblots with R76. Samples from each of the parents of these mutants revealed all three antigenic isoforms of PsNLEC-1 in samples of nodule ho-

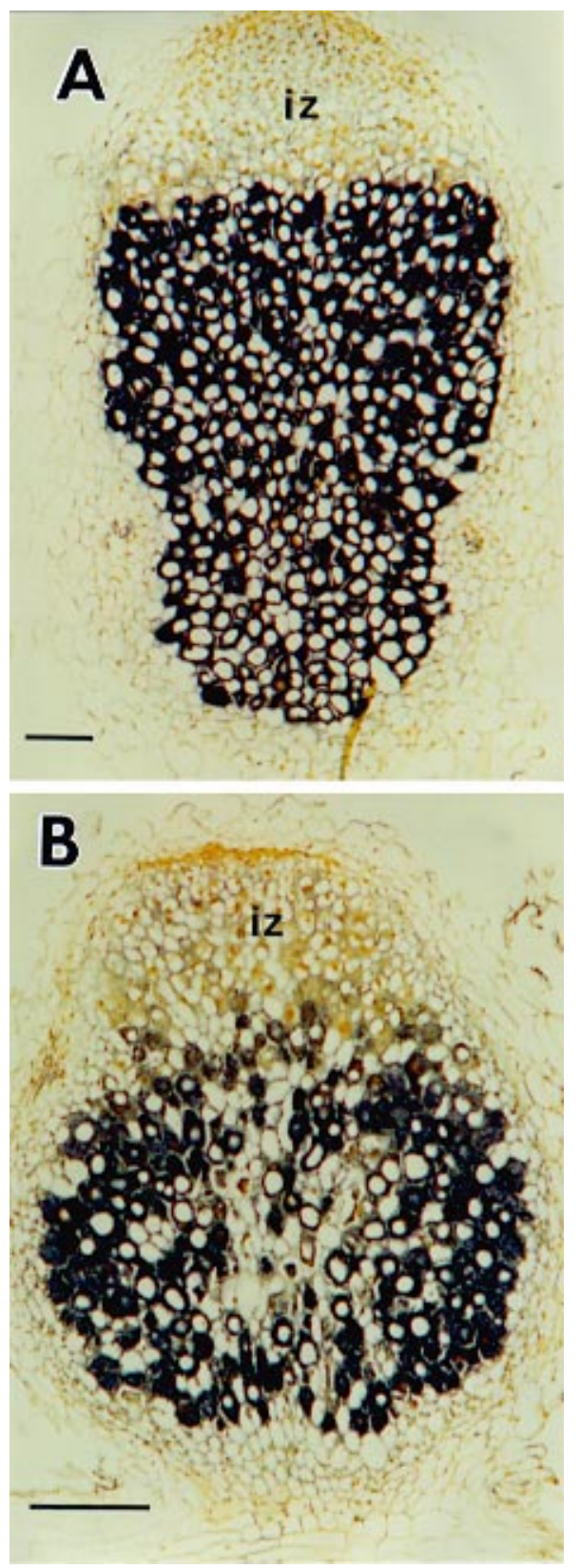

Fig. 4. In situ hybridization in nodule tissue showing expression of PsNlec1 gene transcript as blue-black stain. A, Sprint2; B, sym31, in each case with nodule apex and invasion zone (iz) oriented to the top. Sections were counterstained with Auramine O. Bar $=80 \mu \mathrm{m}$. 
mogenates (data not shown), as had been found previously for Sprint2 and Wisconsin Perfection (Dahiya et al. 1997). However, sym31 was apparently different from all other $\mathrm{Fix}^{-}$mutants examined in lacking the symbiosomal isoforms PsNLEC-1A and B, one or both of which were present in all other Fix ${ }^{-}$mutant pea lines (data not shown).

\section{Expression of PsNLEC-1 antigen and PsNlec1 transcript in tissue sections.}

R76 antiserum was used to localize PsNLEC-1 antigen in longitudinal sections of nodule tissue. Adjacent sections were used for staining with basic fuschin to show general morphology (Fig. 2). In Sprint2 after silver-enhanced immunogold staining (Fig. 3A), the PsNLEC-1 antigen was present throughout the infected tissues in cells containing symbiosomes. The intensity of the label increased as the nodule cells became more mature and was most abundant in the cells at the proximal end of the nodule. Young, infected cells showed hardly any label of PsNLEC-1 antigen. Similarly, there was no evidence of label in the cortex or uninfected parenchyma cells. In the infected cells of sym31 nodules, however, the general level of immunolabeling was much less (Fig. 3B), and it was hard to detect any antigen above the background level obtained with pre-immune serum (data not shown).

In contrast to the evidence from immunogold localization, the results from in situ hybridization studies (Fig. 4) indicated
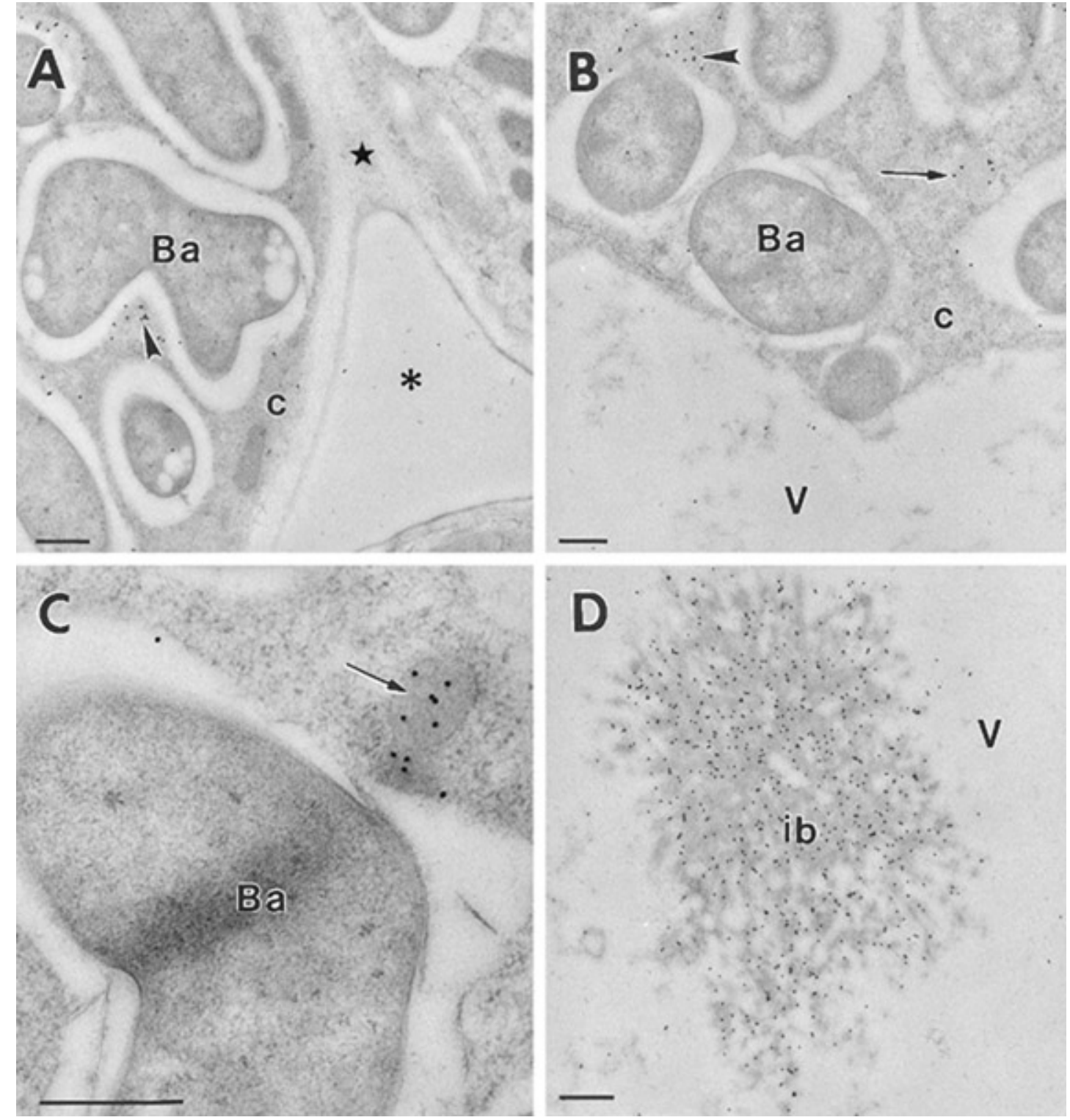

Fig. 5. Electron micrographs of a mature infected cell from a wild-type Sprint 2 nodule, showing immunogold localization of PsNLEC-1 antigen as aggregated protein in peribacteroid space of symbiosomes and as inclusion bodies within vacuole. A, Outer margin of cell, illustrating aggregated antigen (arrowhead) in peribacteroid space adjacent to a bacteroid (Ba) embedded in cytoplasm (c); star and asterisk show, respectively, absence of label from cell wall and intercellular space. B, Central region of a cell, showing small cytoplasmic vesicle carrying PsNLEC-1 antigen (arrow) and vacuole (V) with dispersed antigen. C, Vesicles carrying antigen in close proximity to symbiosomal membrane (arrow). D, Large inclusion body (ib) localized within vacuole of a host cell at proximal end of nodule. Nodule sections were labeled with R76 antiserum followed by $20 \mathrm{~nm}$ of colloidal gold conjugated with anti-rabbit secondary antibody. Bar $=500 \mathrm{~nm}$. 
that PsNlecl transcript was strongly expressed in infected host cells in nodules of mutant and wild type. With this high level of overall expression, there was apparently no gradient of transcript abundance across the infected cells of the central tissue, although PsNLEC-1 antigen only accumulated toward the base of wild-type nodules (Fig. 3A). Expression of transcript in uninfected (interstitial) cells was barely detectable in the central region of wild-type or sym31 nodules (Fig. 4).

\section{Ultrastructural localization of PsNLEC-1 antigen.}

Following immunogold staining of Sprint 2 nodule tissue sections, PsNLEC-1 antigen was mainly localized in the peribacteroid space (PBS) (Fig. 5A,B). Some small cytoplasmic vesicles carrying PsNLEC-1 antigen were also visible (Fig. 5B,C). Pear-shaped vesicles associated with symbiosomes were also observed in sections of osmicated tissues prepared by conventional fixation and embedding techniques (Fig. 6). Although these sections were not used for immunogold staining, the frequency, size, and position of the vesicles were similar to those illustrated in Figure 5C, which appeared to contain PsNLEC-1 antigen. The vacuolar compartment of the infected cells also contained diffused PsNLEC-1 antigen (Fig. 5B). Occasionally, substantial inclusion bodies were observed in the vacuoles of some infected cells at the proximal end of the nodule. These aggregates did not appear to be bound by a membrane and were extensively labeled by colloidal gold (Fig. 5D). No label could be detected in uninfected cells, despite the fact that a low level of PsNlecl transcript had previously been detected by in situ hybridization (Kardailsky et al. 1996).

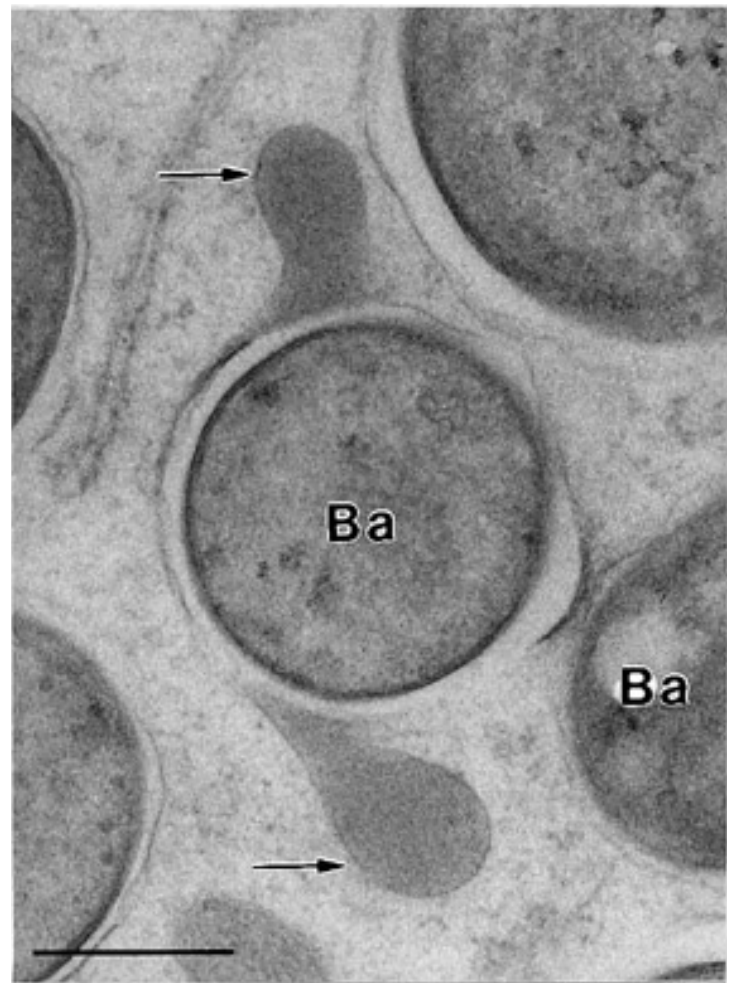

Fig. 6. Ultrathin section of osmium-fixed nodule tisssue showing pearshaped cytoplasmic vesicles in contact with a symbiosome (arrow). Ba, bacteroid. Bar $=500 \mathrm{~nm}$.
In contrast to the wild type, the mutant sym31 did not show any antigen in the symbiosomes nor in the cytoplasm (Fig. 7A). The label was restricted to the vacuole of infected cells, where it was present in the form of inclusion bodies (Fig. 7B, C). These inclusion bodies were different in morphology from those occasionally observed in vacuoles from wild-type nodule cells (Fig. 5D), and most of them were in close proximity to the vacuolar membrane. No label was observed on the cell wall nor in the intercellular space of the nodule cells in either wild-type or mutant tissue (Figs. 5A and 7A). Similarly, there was no label in the uninfected cells or in the parenchymal cells of the Sprint2 and sym 31 nodules. Pre-immune serum did not show any label in any of the nodule tissue, confirming the results obtained with light microscopy (data not shown).

\section{DISCUSSION}

In the present study, we have used PsNLEC-1 antigens as markers for symbiosome differentiation in the symbiotically defective mutant Sprint2Fix ${ }^{-}$(sym31), relative to the wild-type parent line Sprint2. On immunoblots, it was revealed that the mutant did not have any of the antigenic isoforms of PsNLEC1 that are normally associated with symbiosomes (Dahiya et al. 1997). Only the putative vacuolar isoform, PsNLEC-1C, was present (Fig. 1, lane 3). Similarly, the peribacteroid fluid derived from nodule homogenate of sym 31 did not show any band corresponding to PsNLEC-1 antigen (Fig. 1, lane 4). All other pea Fix ${ }^{-}$mutants that were analyzed had either one or both of the symbiosomal isoforms. Mutant sym31 therefore appears to be unusual with respect to the PsNLEC-1 localization, which is consistent with its unusual symbiosome morphology and arrested development (Borisov et al. 1992, 1997; Sherrier et al. 1997).

When median longitudinal sections of Sprint2 nodules were probed with PsNlecl antisense transcript derived from a cDNA clone (Kardailsky et al. 1996), it was found that gene expression was strong and apparently uniform across the infected tissues of the nodule (Fig. 4A). However, when R76 antiserum was used as an immunological probe, the glycoprotein antigen only seemed to be accumulating at the base of the nodule (Fig. 3A). This discrepancy could perhaps indicate that expression from PsNlecl transcript was subject to translational control or, alternatively, that PsNLEC-1 glycoprotein is turned over rapidly in developing symbiosomes and only accumulates in host cells with a positive nitrogen balance, as suggested previously for nodules of Wisconsin Perfection (Dahiya et al. 1997). This latter explanation is consistent with our observations concerning the expression of Cyp 15a, a gene encoding a cysteine protease that is expressed in infected pea nodule tissue (Kardailsky and Brewin 1997) and targeted to the symbiosome compartment (J. L. Vincent and N. J. Brewin, unpublished observations). Furthermore, it has been previously shown that vacuolar legume lectins can turn over very rapidly under some circumstances (Chrispeels and Raikhel 1991). The results for nodules of sym31 indicated that the infected cells of sym 31 also expressed the PsNlecl gene very strongly (Fig. 4B). However, when tissue was probed with R76 at the light microscope level, there was hardly any detectable antigen (Fig. 3B), perhaps suggesting an even higher level of protein turnover in host cells of mutant nodules, compared with Sprint2. 
At the ultrastructural level, immunogold localization in Sprint 2 nodule tissues showed the presence of antigen in the peribacteroid space (PBS) of the symbiosomes (Fig. 5A,B). This localization was essential, similar to that reported previously for the variety Wisconsin Perfection (Dahiya et al. 1997). However, the label was generally more intense for Sprint2 than for Wisconsin Perfection, as was also noted in a previous study (Kardailsky et al. 1996), in which monoclonal antibody MAC266 was used to localize a carbohydrate epitope that is shared between PsNLEC-1 and other Golgiderived glycoprotein components of the peribacteroid fluid. Another interesting difference was that small cytoplasmic vesicles carrying PsNLEC-1 antigen were clearly visible in cells of Sprint2 (Fig. 5B,C). In nodule tissue fixed with $\mathrm{OsO}_{4}$ for better preservation of the membrane, pear-shaped vesicles (approximately $200 \mathrm{~nm}$ in diameter) were observed in contact with the symbiosomal membrane (Fig. 6). It seems probable that these vesicles fuse with the symbiosome membrane, thereby delivering PSNLEC-1 antigen into the symbiosomal space. Previous ultrastructural studies have revealed similarsized vesicles in association with the symbiosomal membranes of pea and soybean nodule cells (Kijne and Planque 1979). However, in view of their size, it is unlikely that these vesicles are derived directly from the Golgi body; more probably they form part of a pre-vacuolar compartment that has recently been identified (Paris et al. 1996, 1997). It is interesting to note that rather similar aspherical or "pyriformis" vesicles (50 to $100 \mathrm{~nm}$ in diameter) have previously been observed by Ridge (1988), following freeze-substitution of root hair cells of Vicia hirsuta; these vesicles were concentrated at the extreme tip of the root hair cell, a region of intense secretory activity that, in pea, has also been associated with the targeted secretion of pea seed lectin antigen (Díaz et al. 1995). In addition, the vacuoles of a few mature, infected cells in Sprint 2 nodules contained large, aggregated protein bodies extensively labeled by colloidal gold (Fig. 5D). Localization of a legume lectin antigen in both the symbiosomal and vacuolar compartments has been previously reported for nodules of other species, e.g., peanut (VandenBosch et al. 1994), but the possible relationship between symbiosome development and the mechanism of protein body formation in pea cotyledons (Hinz et al. 1995) has not been systematically explored.

In sym31, there was no evidence of immunogold label in the symbiosomes nor in the cytoplasm (Fig. 7A), which is consistent with the observed absence of isoforms PsNLEC-1A and $1 \mathrm{~B}$ from the nodule homogenates of sym31 on Western blots. The only site of accumulation of antigen was in the vacuoles of some host cells where heavily labeled inclusion bodies were observed (Fig. 7B,C). Interestingly, these inclusion bodies were different with respect to number, shape, position, and compactness, relative to those observed in host cells of Sprint2, perhaps reflecting differences in the way that

Fig. 7. Electron micrographs of sym31 nodule cells, showing distribution of R76 antigen after immunogold staining. A, Infected cell, showing absence of label from symbiosomes and cytoplasm (c); star and asterisk show absence of label from cell wall and intercellular space, respectively. B, Inclusion bodies (ib) in vacuole (V), showing extensive labeling. C, Enlargement of central inclusion body illustrated in B. Bar $=1.0 \mu \mathrm{m}$. they were formed. In the wild-type (Fig. 5D), the vacuolar inclusion body was large and diffuse, suggesting that it had been formed by protein aggregation within the vacuolar compartment. On the other hand, in sym31 (Fig. 7B,C), the smaller
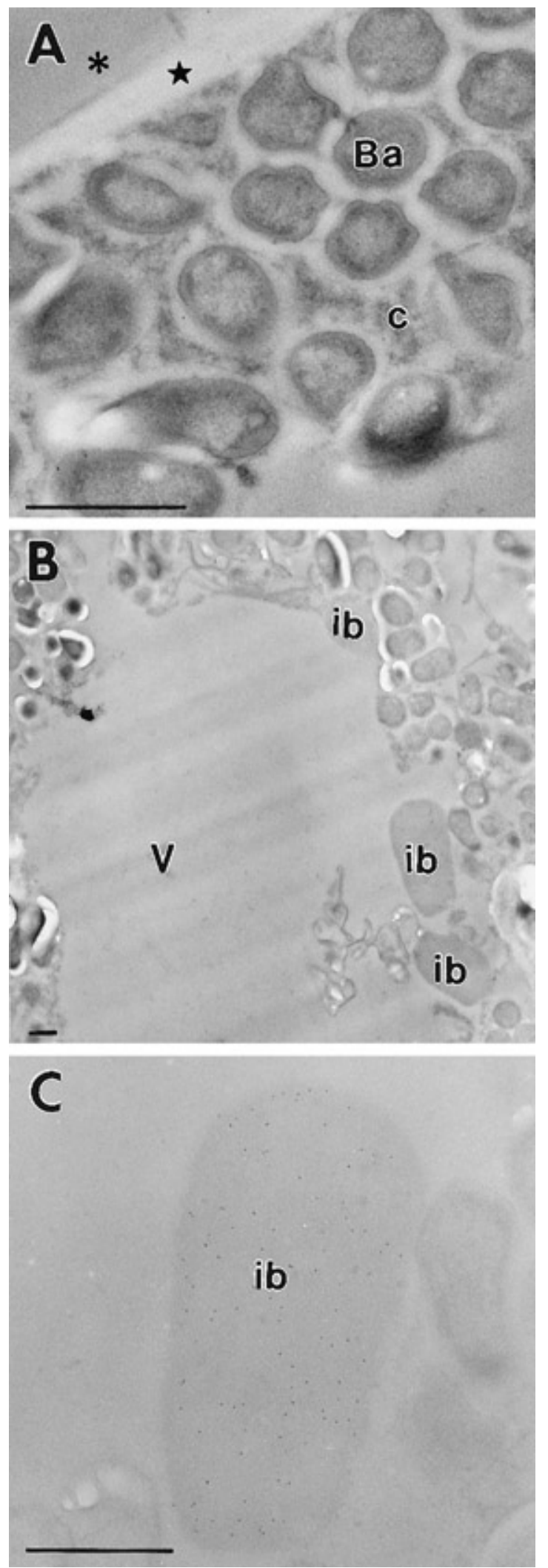
and more numerous inclusion bodies were usually distributed close to the cytoplasmic margins of the vacuole, suggesting that the PsNLEC-1 antigen may have accumulated in a prevacuolar body that had subsequently discharged its contents by fusion with the tonoplast membrane (Paris et al. 1997).

The observed absence of PsNLEC-1 from symbiosomes of sym31 could be explained in several ways. One possible explanation is that sym 31 is defective in vesicle targeting from the Golgi to the symbiosome compartment. In this model, the post-Golgi vesicles that would normally be targeted to the symbiosomes are instead targeted to the vacuole: it is an open question whether the observed absence of "symbiosomal" isoforms PsNLEC-1A and PsNLEC-1B might be a cause or a consequence of mis-targeting of vesicles. Various scenarios can be imagined, but we are currently unable to distinguish between these possibilities. One alternative explanation could be that targeting is normal but all of the PsNLEC-1 in the symbiosome compartment is turned over very rapidly in the mutant and therefore antigen is undetectable by immunolabeling. But this explanation fails to account for the observation from immunogold localization that vacuoles of mutant cells appear to have a larger quantity of the PsNLEC-1 protein than vacuoles of newly infected wild-type host cells, which are at a comparable stage of symbiosome development. By contrast, in the wild type, PsNLEC-1 antigen starts accumulating in the vacuole only when cells become very mature. Rapid autolysis of symbiosomes has been proposed for the symbiotically defective pea mutant sym13 (Kneen et al. 1990a) but the morphology of symbiosomes and bacteroids is entirely different for this mutant, compared with sym31 (Borisov et al. 1997). In sym31, the development of the bacteroids and symbiosomal membrane is arrested at an early stage (Sherrier et al. 1997), corresponding to the developmental equivalent of newly infected wild-type cells in the early symbiotic zone. These unique ultrastructural features tend to support the interpretation that PsNLEC-1 protein is targeted exclusively to the vacuoles in the host cells of sym31. We postulate that a symbiosome-specific vesicle targeting pathway may have failed to differentiate during nodule development. It is interesting that vesicles containing PsNLEC-1 antigen are not apparently targeted to the exterior of the cell that is the normal default pathway for vesicles not carrying specific signals (Bednarek and Raikhel 1992). Targeting of legume lectin gene products to the vacuole is a common occurrence for many seed and vegetative lectins (Hinz et al. 1995) although for some legume lectins an extracellular location has also been observed (VandenBosch et al. 1994; Kjemtrup et al. 1995).

In conclusion, on the basis of evidence from cell fractionation and immunolabeling studies, we have shown that almost all of the PsNLEC-1 antigen in nodules of Sprint2Fix ${ }^{-}$ (sym31) seems to correspond to isoform $\mathrm{C}$ and to be localized in the vacuole. By implication, therefore, the symbiotic gene Sym31 controls (directly or indirectly) the presence of PsNLEC-1 in the symbiosome compartment. This study of a symbiotically defective mutant has apparently revealed a defective targeting system for the PsNLEC-1 protein, but it is still not clear how this defect in symbiosome development correlates with the Fix ${ }^{-}$phenotype, nor with the abnormal carbon metabolism of this mutant (Romanov et al. 1995). Further studies with PsNlecl transcript and R76 antiserum as molecular probes could help to elucidate important developmental pathways of the endomembrane system in nodule cells.

\section{MATERIAL AND METHODS}

\section{Biological material.}

Samples of Pisum sativum variety Sprint 2 and mutant derivative Sprint2Fix ${ }^{-}$(sym31) were obtained as previously described (Borisov et al. 1992; Sherrier et al. 1997). For pea nodulation, seedlings were inoculated with the Rhizobium leguminosarum bv. viciae wild-type strain 3841 (Wood et al. 1989). Plants were grown in gnotobiotic conditions in conical flasks on agar medium containing mineral salts but lacking a nitrogen source. Samples of $\mathrm{Fix}^{-}$mutants E135f (sym13) (Kneen et al. 1990a), FN1 (Postma et al. 1990), E107 (Kneen et al. 1990b) and P63 (sym26) (Sagan et al. 1993a, 1993b) together with corresponding wild-type lines (Sparkle, Rondo, and Frisson) were obtained from the pea germplasm collection at the John Innes Centre (courtesy of Mike Ambrose).

Rabbit antiserum R76, reacting with PsNLEC-1 antigen, was prepared as previously described (Dahiya et al. 1997). Antigenic material was obtained by heterologous expression of the PsNlec1 cDNA cloned in Escherichia coli. The antiserum was routinely precipitated with $E$. coli lysate to remove cross-reacting antibodies. For immunogold staining, R76 was further purified by immunoadsorption to heterologous PsNLEC-1 protein expressed in E. coli, as previously described (Dahiya et al. 1997). Rat monoclonal antibody MAC254 reacts with carbohydrate epitopes associated with glycoproteins (including PsNLEC-1) that are targeted to the peribacteroid fluid (Perotto et al. 1991).

\section{Fractionation of pea nodule homogenate.}

A simple sucrose step gradient was used for the isolation of symbiosomal fluid from pea nodule homogenate (Brewin et al. 1985; Dahiya et al. 1997). All procedures were carried out at $4^{\circ} \mathrm{C}$, in the presence of a cocktail of protease inhibitors (Boehringer, Mannheim, Germany): p-amino benzamidine (10 $\mu \mathrm{g} / \mathrm{ml})$, 4-amidino-phenyl-sulfonyl fluoride (APMSF) (10 $\mu \mathrm{g} / \mathrm{ml})$, phosphoramidon $(1 \mu \mathrm{g} / \mathrm{ml})$, E64 $(1 \mu \mathrm{g} / \mathrm{ml})$, and pepstatin $(1 \mu \mathrm{g} / \mathrm{ml})$. Nodules, 3 to 4 weeks post-infection, were collected in the extraction buffer $(50 \mathrm{mM}$ Tris- $\mathrm{HCl}, 10 \mathrm{mM}$ dithiothreitol [DTT], $500 \mathrm{mM}$ sucrose, $\mathrm{pH}$ 7.4) and were homogenized with a mortar and pestle in the presence of insoluble polyvinylpolypyrrolidone (Sigma, Poole, UK). The extract was filtered through two layers of Miracloth. The symbiosomes and the nodule supernatant were separated by centrifugation in microcentrifuge tubes for $1 \mathrm{~min}$ at $10,000 \times g$. The symbiosomal material was released from intact symbiosomes by osmotic shock and mechanical force in the form of vortexing and pipetting. Released bacteroids were removed by centrifugation at $10,000 \times g$ for $2 \mathrm{~min}$. The symbiosomal material was further fractionated by centrifugation at $100,000 \times g$ for $30 \mathrm{~min}$ to separate the symbiosomal fluid (peribacteroid fluid) and the symbiosomal membrane (peribacteroid membrane) fractions.

\section{Gel electrophoresis and Western blotting.}

Samples were separated by sodium dodecyl sulfatepolyacrylamide gel electrophoresis (SDS-PAGE) (Laemmli 
1970) with $12 \%$ (wt/vol) polyacrylamide minigels. Equal weights of frozen nodules $(1 \mathrm{mg})$ were ground in solubilization buffer and boiled immediately to protect the PsNLEC-1 antigen from being destroyed by protease activity. For mutants, the total volume of nodule homogenate loaded on gels was five times more than for parent lines, to compensate for the lower quantity of total protein present. For nodule homogenates, $10 \mu \mathrm{g}$ of protein was loaded per well and for peribacteroid fluid protein loading was $50 \mathrm{ng}$ per well. Equal loading of samples was confirmed by staining with Coomassie brilliant blue with silver ions. It was shown by immunostaining with MAC254 (Perotto et al. 1991) that the symbiosomal fluid derived from sym 31 nodule homogenates contained the expected plant glycoprotein antigens recognized by this antibody although the yield of peribacteroid fluid was very low in this mutant (data not shown).

For Western blotting, antigens were transferred electrophoretically to nitrocellulose membranes (Schleicher and Schuell, Dassel, Germany), with $40 \mathrm{~V}$ constant voltage for $1 \mathrm{~h}$ at room temperature. The transfer buffer was $0.1 \mathrm{mM}$ CAPS (3-[cyclohexylamino]1-propane sulfonic acid) (obtained from Sigma, Poole, UK) pH 10.5 with $10 \%$ (vol/vol) methanol.

\section{Microscopy.}

Pea nodules were fixed and embedded in London Resin White (London Resin, Basinstoke, U.K.) as described previously (VandenBosch et al. 1989). The general anatomy of thick sections was revealed by staining with basic fuscin. Gold sections, 90 to $150 \mathrm{~nm}$ thick, were collected for immunogold labeling on gold grids coated with $4 \%$ (wt/vol) parlodion and carbon. The sections were incubated overnight at $4^{\circ} \mathrm{C}$ with primary antibody followed by secondary anti-rabbit antibody conjugated to $20 \mathrm{~nm}$ colloidal gold (Amersham International, Amersham, UK). The sections were counterstained in $2 \%(\mathrm{wt} / \mathrm{vol})$ aqueous uranyl acetate for $15 \mathrm{~min}$. Osmicated tissue samples were fixed as described by Sherrier et al. (1997). Nodule tissues were viewed and photographed in a transmission electron microscope (JEOL JEM$1200)$ at $80 \mathrm{kV}$. Silver enhancement was carried out as described previously (VandenBosch 1992), with materials obtained from Amersham.

Longitudinal sections of wax-embedded pea nodule tissue were used for in situ hybridization studies. Digoxigeninlabeled riboprobes were synthesized with linearized plasmid that contained the 900 bp 3' rapid amplification cDNA ends (RACE) product of PsNlecl (Kardailsky et al. 1996) subcloned in sense and antisense orientation behind the T7 promoter of the pBluescript vector SK(+). Nodules were fixed and sectioned as described previously (De Block and Debrouwer 1992; Gardner et al. 1996). Sections were hybridized with riboprobes and the signal was visualized with alkaline phosphatase. Auramine $\mathrm{O}$ was used as counterstain.

\section{ACKNOWLEDGMENTS}

P. D. was supported by the Commonwealth Commission; D. J. S. by the BBSRC Intracellular Signalling Programme; I. V. K. by the EU Biotechnology Inco-Copernicus programme (CIPA-CT93-0156); A. Y. B. by the Russian Foundation for Basic Research (96-04-50361, 9704-50033); and N. J. B. by core funding from BBSRC to the John Innes Centre.

\section{LITERATURE CITED}

Bednarek, S. Y., and Raikhel, N. V. 1992. Intracellular trafficking of secretory proteins. Plant Mol. Biol. 20:133-150.

Borisov, A. Y., Morzina, E. V., Kulikova, O. A., Tchetkova, S. A., Lebsky, V. K., and Tikhonovich, I. A. 1992. New symbiotic mutants of pea (Pisum sativum L.) affecting either nodule initiation or symbiosome development. Symbiosis 14:297-313.

Borisov, A. Y., Rosov, S. N., Tsyganov, V. E., Morzhina, E. V., Lebsky, V. K., and Tikhonivich, I. A. 1997. Sequential functioning of Sym-13 and Sym-31, two genes affecting symbiosome development in root nodules of pea (Pisum sativum L.). Mol. Gen. Genet. 254:592-598.

Brewin, N. J. 1991. Development of the legume root nodule. Annu. Rev. Cell Biol. 7:191-226.

Brewin, N. J., and Kardailsky, I. V. 1997. Legume lectins and nodulation by Rhizobium. Trends Plant Sci. 2:92-98.

Brewin, N. J., Robertson, J. G., Wood, E. A., Wells, B., Larkins, A. P., Galfrè, G., and Butcher, G. W. 1985. Monoclonal antibodies to antigens in the peribacteroid membrane from Rhizobium-induced root nodules of pea cross-react with plasma membrane and Golgi bodies. EMBO J. 4:605-611.

Chrispeels, M. J., and Raikhel, N. V. 1991. Lectins, lectin genes and their role in plant defense. Plant Cell 3:1-9

Dahiya, P., Kardailsky, I. V., and Brewin, N. J. 1997. Immunolocalization of PsNLEC-1, a lectin-like glycoprotein expressed in developing pea nodules. Plant Physiol. 115:1431-1442.

De Block, M., and Debrouwer, D. 1992. In-situ enzyme histochemistry on plastic-embedded plant material. The development of an artefactfree beta-glucuronidase assay. Plant J. 2:261-266.

Díaz, C. L., Logman, T. J. J., Stam, H. C., and Kijne, J. W. 1995. Sugarbinding activity of pea lectin expressed in white clover hairy roots. Plant Physiol. 109:1167-1177.

Faye, L., Johnson, K. D., Storm, A., and Chrispeels, M. J. 1989. Structure, biosynthesis and function of asparagine-linked glycans on plant glycoproteins. Physiol. Plant. 75:309-314.

Gardner, C. D., Sherrier, D. J., Kardailsky, I. V., and Brewin, N. J. 1996. Localization of lipoxygenase proteins and mRNA in pea nodules: Identification of lipoxygenase in the lumen of infection threads. Mol. Plant-Microbe Interact. 9:282-289.

Hinz, G., Hoh, B., Hohl, I., and Robinson, D. G. 1995. Stratification of storage proteins in the protein storage vacuole of developing cotyledons of Pisum sativum L.. J. Plant Physiol. 145:437-442.

Kardailsky, I. V., and Brewin, N. J. 1997. Expression of cysteine protease genes in pea nodule development and senescence. Mol. PlantMicrobe Interact. 9:689-695.

Kardailsky, I. V., Sherrier, D. J., and Brewin, N. J. 1996. Identification of a new pea gene, PsNlec1, encoding a lectin-like glycoprotein isolated from the symbiosomes of root nodules. Plant Physiol. 111:49-60.

Kijne, J. W. and Planqué, K. 1979. Ultrastructural study of the endomembrane system in infected cells of pea and soybean root nodules. Physiol. Plant Pathol. 14:339-345.

Kjemtrup, S., Borkhsenious, O., Raikhel, N. V., and Chrispeels, M. J. 1995. Targeting and release of phytohemagglutinin from the roots of bean seedlings. Plant Physiol. 109:603-610.

Kneen, B. E., LaRue, T. A., Hirsch, A. M., Smith, C. A., and Weeden, N. F. 1990a. sym13 - a gene conditioning ineffective nodulation in Pisum sativum. Plant Physiol. 94:899-905.

Kneen, B. E., LaRue, T. A., Welch, R. M., and Weeden, N. F. 1990b. Pleiotropic effects of $\mathrm{brz}^{l}$ : a mutation in Pisum sativum (L.) cv 'Sparkle' conditioning decreased nodulation and increased iron uptake and leaf necrosis. Plant Physiol. 93:717-722.

Laemmli, U. K. 1970. Cleavage of structural proteins during the assembly of the head of bacteriophage T4. Nature 227:680-685.

Mylona, P., Pavlowski, K., and Bisseling, T. 1995. Symbiotic nitrogen fixation. Plant Cell 7:869-885.

Paris, N., Rogers, S. W., Jiang, L., Kirsch, T., Beevers, L., Phillips, T. E., and Rogers, J. C. 1997. Molecular cloning and further characterization of a probable plant vacuolar sorting receptor. Plant Physiol. 115: 29-39.

Paris, N., Stanley, C. M., Jones, R. L., and Rogers, J. C. 1996. Plant cells have two functionally distinct vacuolar compartments. Cell 85:563-572.

Perotto, S., Donovan, N., Drobak, B. K., and Brewin, N. J. 1995. Differential expression of a glycosyl inositol phospholipid antigen on the peribacteroid membrane during pea nodule development. Mol. Plant- 
Microbe Interact. 8:560-568.

Perotto, S., VandenBosch, K. A., Butcher, G. W., and Brewin, N. J. 1991. Molecular composition and development of the plant glycocalyx associated with the peribacteroid membrane of pea root nodules. Development 112:763-773.

Postma, J. G., Jager, D., Jacobsen, E., and Feenstra, W. J. 1990. Studies on a non-fixing mutant of pea (Pisum sativum L.) I. Phenotypical description and bacteroid activity. Plant Sci. 68:151-161.

Ridge, R. W. 1988. Freeze substitution improves the ultrastructural preservation of legume root hairs. Bot. Mag. Tokyo 101:427-441.

Romanov, V. I., Gordon, A. J., Minchin, F. R., Witty, J. F., Skot, L., James, C. L., Borisov, A. Y., and Tikhonovich, I. A. 1995. Anatomy, physiology and biochemistry of root nodules of Sprint-2Fix(-), a symbiotically defective mutant of pea (Pisum sativum L.). J. Exp. Bot. 46: 1809-1816.

Sagan, M., Huguet, T., Barker, D. G., and Duc, G. 1993a. Characterization of two classes of non-fixing mutants of pea plants (Pisum sativum L.). Plant Sci. 95:55-66.

Sagan, M., Messager, A., and Duc, G. 1993b. Specificity of the Rhizobium-legume symbiosis obtained after mutagenesis in pea (Pisum sativum L.). New Phytol. 125:757-761.

Sherrier, D. J., Borisov, A. Y., Tikhonovich, I. A., and Brewin, N. J 1997. Immunocytological evidence for abnormal symbiosome development in nodules of the pea mutant line Sprint2Fix ${ }^{-}$(sym31). Proto- plasma 199:57-68

VandenBosch, K.A. 1992. Localization of proteins and carbohydrates using immunogold labelling in light and electron microscopy. Pages 31-43 in: Molecular Plant Pathology, A Practical Approach. Vol. 2. S. J. Gurr, M. J. McPherson, and D. J. Bowles, eds. Oxford University Press, Oxford.

VandenBosch, K. A., Bradley, D. J., Knox, J. P., Perotto, S., Butcher, G. W., and Brewin, N. J. 1989. Common components of the infection thread matrix and the intercellular space identified by immunocytochemical analysis of pea nodules and uninfected roots. EMBO J. 8: 335-342.

VandenBosch, K. A., Rodgers, L. R., Sherrier, D. J., and Kishinevsky, B. D. 1994. A peanut nodule lectin in infected cells and in vacuoles and the extracellular matrix of nodule parenchyma. Plant Physiol. 104: 327-337.

Vasse, J., de Billy, F., Camut, S., and Truchet, G. L. 1990. Correlation between ultrastructural differentiation of bacteroids and nitrogen fixation in alfalfa nodules. J. Bacteriol. 172:4295-4306.

Verma, D. P. S., and Hong, Z. L. 1996. Biogenesis of the peribacteroid membrane in root nodules. Trends Microbiol. 4:364-368.

Wood, E. A., Butcher, G. W., Brewin, N. J., and Kannenberg, E. L. 1989. Genetic derepression of a developmentally regulated lipopolysaccharide antigen from Rhizobium leguminosarum 3841. J. Bacteriol. 171: $4549-4555$ 